\title{
Differential Gene Expression Associated with BMI, Gender, and IBS-subtype in Human White Blood Cells: Results from a Custom 250-plex Nanostring Probe Panel.
}

Robinson JM

Email: jeff.robinson.evol@gmail.com

Linkedln: https://www.linkedin.com/in/robinsonjmevol/

ResearchGate: https://www.researchgate.net/profile/Jeffrey Robinson4

\begin{abstract}
$\underline{\text { Abstract }}$
Chronic gastro-abdominal pain with altered bowel habits are associated with pathologies including gastroenteritis, autoimmune and inflammatory bowel disease, and irritable bowel syndrome (IBS). In IBS, diagnostic evidence of infection or inflammation are absent, yet symptoms nonetheless include chronic abdominal pain and alterations of stool frequency and consistency, with most common subtypes including diarrhea-predominant (IBS-D) or constipation predominant (IBS-C). [1, 2] IBS is a common clinical complaint in westernized nations, with females more frequently diagnosed than males [3]. Obesity is also associated with increased likelihood of chronic pain [4], and is associated with intestinal dysbiosis, and systemic inflammatory signatures [5]. Improving personalized medicine therefore requires patient stratification based on a combination of biological factors contributing to the individual's symptom and biomarker spectrum. The "buffy coat" method isolates leukocytes and PBMCs from whole blood, comprises the immune cell population in peripheral blood. These cells are relatively inactive but are primed to respond to generalized and localized immune activation signals; they are under active investigation as diagnostic biomarkers [6]. Collection of buffy coat is a standard method in clinical laboratories; detection of immune activation via RNA expression biomarkers will be informative of differential biological response, and be tractable to collect in clinical settings. Results of differential expression, pathway analysis, and cell-type abundance analysis from buffy coat RNA, is presented here using variables for BMI, Gender, and IBS-subtype from publicly available Nanostring RNA expression and phenotypic data from the NCBI GEO database.
\end{abstract}

Keywords: IBS; BMI; Nanostring; White Blood Cells; RNA expression

\section{Introduction}

This brief report describes differential expression, quantitative pathway enrichment, and cell-abundance results from human PBMC samples for criteria of BMI, IBS-subtype, and gender from human buffy coat samples. The analyses utilizes publicly available data obtained from the NCBI GEO database series GSE12459 (https://www.ncbi.nlm.nih.gov/geo/query/acc.cgi?acc=GSE124549), and BioProject PRJNA512473 (ID: 512473) (https://www.ncbi.nlm.nih.gov/bioproject/PRJNA512473), and freely available Nanostring software.

A previous report (Robinson et al. 2019) [7] utilized complete blood count with differential (CBC-D) data, with serum Cortisol and other ELISA biomarkers, and reported on associations between IBS subtype, weight, and Cortisol level from this cohort. However, the analysis utilized a categorical weight variable classifying the entire population as either "Normal" (BMI <= 25) or "Overweight" (BMI > 25), criteria which are unable to resolve the effects of continuously increasing BMI, particularly separation of the categories of "Overweight" (BMI > 25 AND $<30)$ and "Obese" (BMI >=30), which is likely to have major effects as a physiological parameter. An updated analysis utilizing the same data was performed during development of R-language script "AnalyzeBloodwork v1.0" (https://github.com/PhyloGrok/AnalyzeBloodwork) [8]. Results subsequently showed, using multiple linear regression analysis, that $\mathrm{BMI}$, Cortisol, and C-Reactive Protein (CRP, a generic inflammatory marker) exhibit a statistically significant 3-way linear relationship.

The current results do not include every subject reported in (Robinson et al. 2019), but complete CBC-D results for each sample reported here may be found in the in the NCBI GEO database for further analysis. Here, results from differential RNA expression are tested with 3 relevant parameters: 
1. IBS subtype. Modeled as categorical independent variable, with separate tests for IBS-D and IBS-C relative to controls, respectively. It must be stated that the control population is significantly larger than either IBS subtype, which may influence the results of statistical tests.

2. BMI. DE tested with a linear model from continuous BMI data.

3. Gender. DE tested (arbitrarily) for female relative to male.

\section{Methods}

GEO series GSE12459 reports that buffy-coat samples were collected using Lympoprep density gradient protocol, total RNA was extracted using the miRVana kit. This analysis includes RNA expression data from 97 samples obtained from an adult population of mixed gender with a subset of individuals with IBS-D or IBS-C diagnosis.

The Nanostring 250-plex custom probe panel "ImmunoGC" is accessioned, with probe sequences, in the GEO Platform GPL25966 (https://www.ncbi.nlm.nih.gov/geo/query/acc.cgi?acc=GPL25996). Additional probe panel annotation data is provided here as well (Sup. File 1: Probe Panel Annotations). The probe panel is significantly enriched for glucocorticoid-responsive immune genes, with citations provided in the supplementary file 1 [9-14]. The GSE12459 description lists the probe panel as an ad-hoc panel that includes probes for genes "1) reported glucocorticoid immune responsiveness, 2) immune-cell type specific expression, 3) microRNA processing, and 4) putative stress-associated biomarkers."

Nanostring methodology is a multiplexed, direct-count method utilizing fluorescent bar-code probes labels with automated library preparation and optical counting which does not require reverse-transcription or amplification of RNA samples [15].

Analysis was performed using open-source software Nanostring nSolver 3.0, and Nanostring Advanced Analysis Module $2.0[16,17]$. Advanced analysis module includes a relative cellular abundance assay based on cell-type specific genes, utilizing a validated, published algorithm [18]. Similar algorithms have been developed and implemented independently, and are increasingly common for cell-type deconvolution (particularly for immune cells) in heterogeneous samples [19-23].

\section{Results and Discussion}

Results presented below represent a selection of results the Nanostring Advanced Analysis Module 2.0 output. The original output is html-formatted, and the complete results are provided here as .zip format files with the complete results accessible via the html main menu and in the sub-directories (Sup. File 2: IBS-subtype analysis; Sup. File 3: BMI analysis; Sup. File 4: Gender analysis).

IBS-subtype. In the first analysis, IBS-D and IBS-C were compared to healthy controls (Fig. 1A). No signal between these groups was seen in the PCA analysis (Fig. 1B). However, several genes were differentially expressed in IBSD vs. controls at $p<.05$ (Fig. 1C, Sup.2) including IL1rn, TLR2, and others, and in IBS-C vs. Control including IL-6 (Sup. File 2). In the gene set enrichment results, decreased pathway enrichment was observed in "Degradation of extracellular matrix" and "Metabolism of carbohydrates" in both IBS-D and IBS-C vs. controls, and "RAFindependent MAPK13 activation" specifically in IBS-C vs Controls (Fig. 1D, Sup. 2). Immune-cell abundance analysis shows an apparent decrease in Neutrophils, Macrophages (Monocytes), and Dendritic Cells (Fig. 1E, Sup. 2). While gene-specific signal from these cells did not reach significance threshold, the decrease in Macrophage abundance corresponds with observations previously reported from the CBC-D monocyte results from the same cohort (Robinson et al. 2019).

BMI. The second analysis utilized BMI as a continuous variable, using a linear model to assess differential expression. The distribution of subject BMI's is provided, once again BMI 18.5-25 is within normal range, 25-30 indicates overweight, while $>30$ indicates obese (Fig. 2A, Sup. 3). The PCA analysis does not readily identify a 
strong signal for this variable (Fig. 2B, Sup. 3). Differential expression shows several significantly increased mRNAs including TGFB2, SAAS, IL6, CD9, LTK, and F13A1. Interestingly, IL1rn shows decreased expression, similar to that seen in IBS-D vs. Controls test (Fig. 2C, Sup. 3). Significantly, in BMIs above 35, a clear trend is seen with decreased cell abundance signal from Neutrophils, DC's, AND Macrophages (Fig. 2D, 2E, Sup. 3), quite similar to the pattern observed with IBS-D vs. Controls. This may reflect that that obese subjects are more common in the IBS-D cohort, and therefore the cell-type signal from IBS-D is influenced by the BMI variable, although it may also reflect an overlapping cellular response mechanistic commonality between obesity and IBS-D.

Gender. Distribution between genders in the cohort was approximately equal (Fig. 3A). In this case, the $3^{\text {rd }}$ principal component (ie. \%8 of variation in the dataset), differentiated the overall signal between male and female (Fig. 3B). Despite the clear differentiation of genders by the PCA analysis, only 3 mRNAs showed differential expression at $p>.05$. These included (in females vs. baseline of male) increased in MME, IL1rn (which interestingly has lower expression in BMI AND IBS), FPR2, and lowered CEBPB expression. (Fig. 3C)

Discussion. These three analyses provide some preliminary results for analysis of the GEO GSE12459 Nanostring data series. They may be further used in metadata compilations and as a comparison with RNA-seq data from the many studies which include gender and BMI variables. In understanding the interplay between weight, obesity, GI pain and gender in personalized medicine, the necessity for addressing these (and other potentially confounding variables such as race) will provide increasing precision for individualized diagnostic strategies. 


\section{Figure 1. IBS-subtype analysis}

A. Sample Distributions

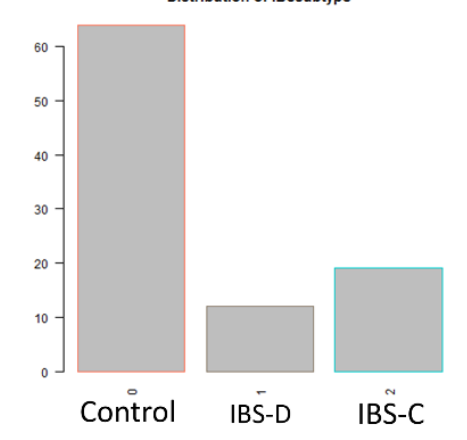

B. PCA: IBS Subtype

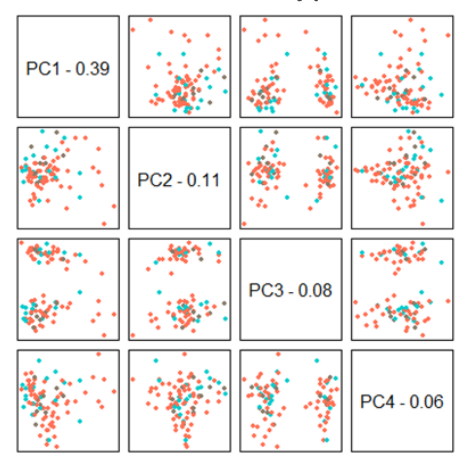

C. DE: IBS-D vs Control

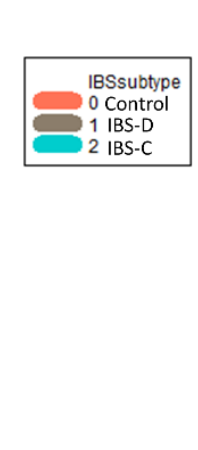

D. Relative Pathway Enrichment

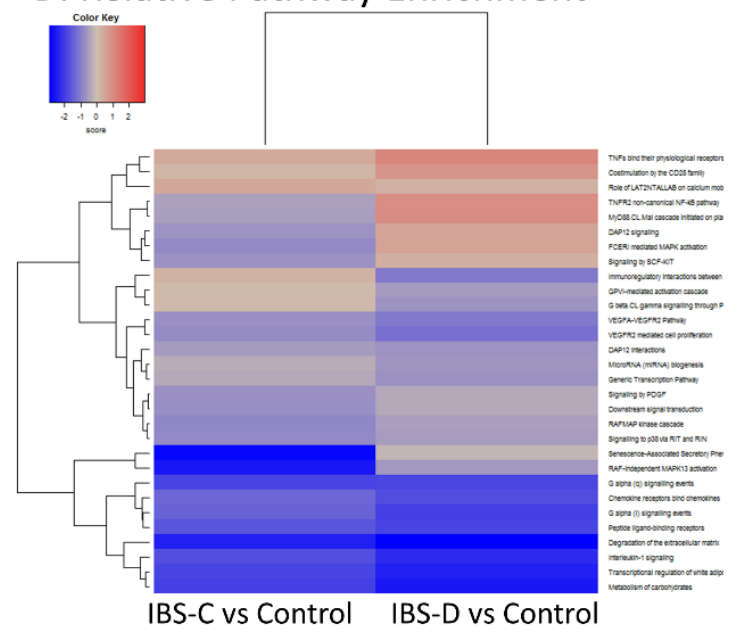

E. Cell-type Scores: IBS subtypes

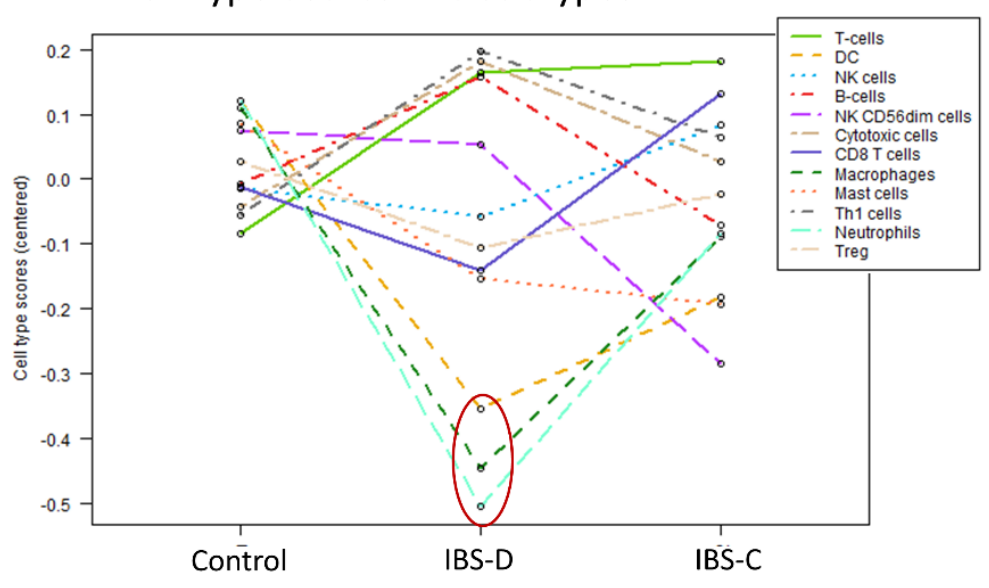

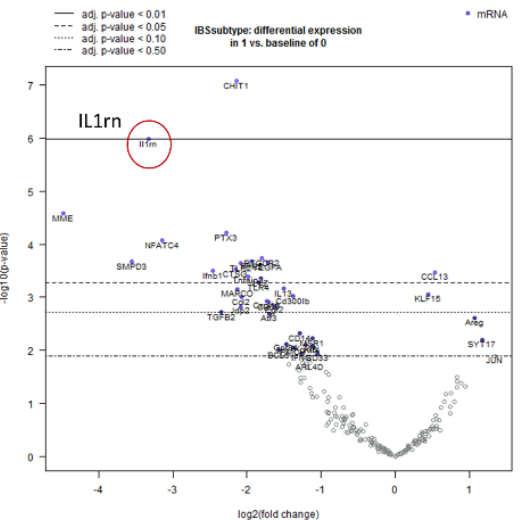




\section{Figure 2. BMI Analysis}

A. Sample Distribution

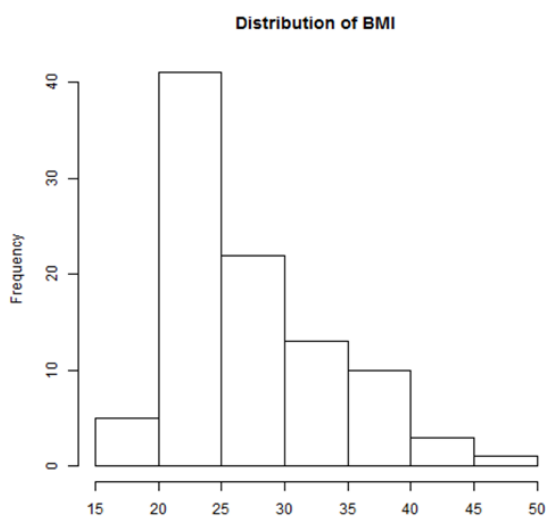

D. Cell-type abundance by BMI

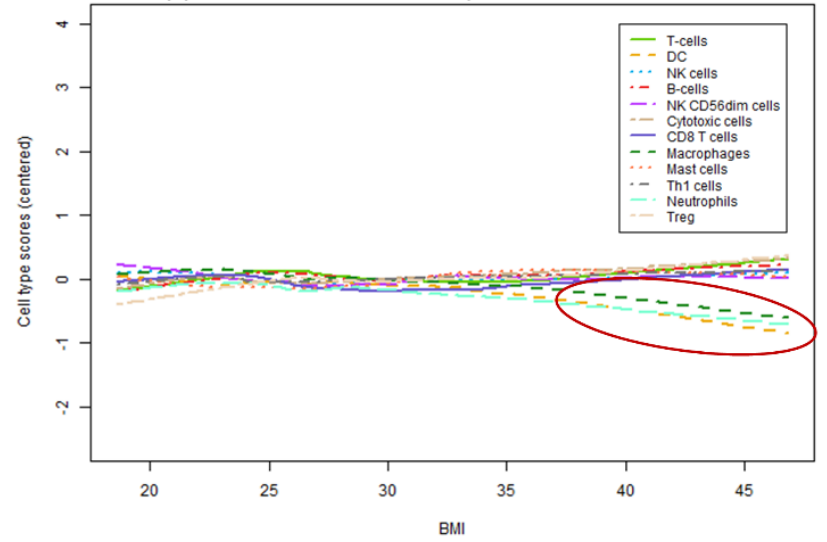

C. DE: BMI linear variable

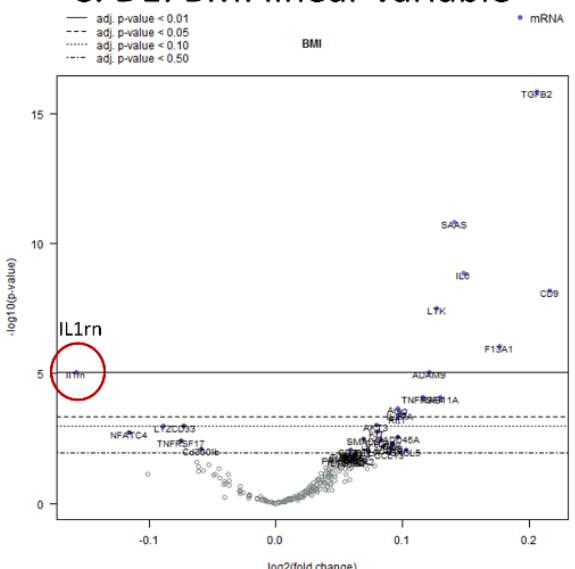

E. Macrophage (ie. Monocyte) score by BMI

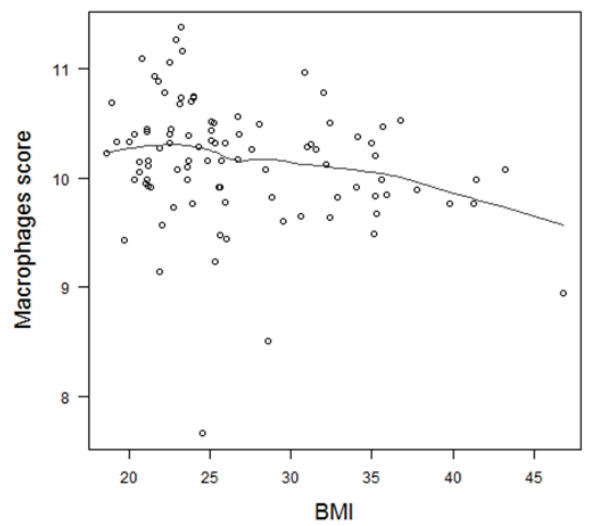




\section{Figure 3. Gender Analysis}

\section{A. Gender Distribution}

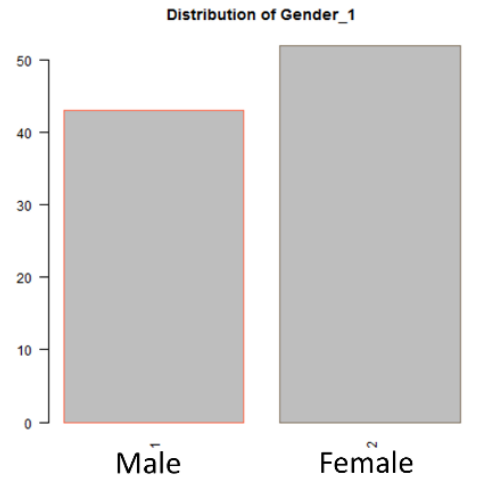

B. PCA: Gender
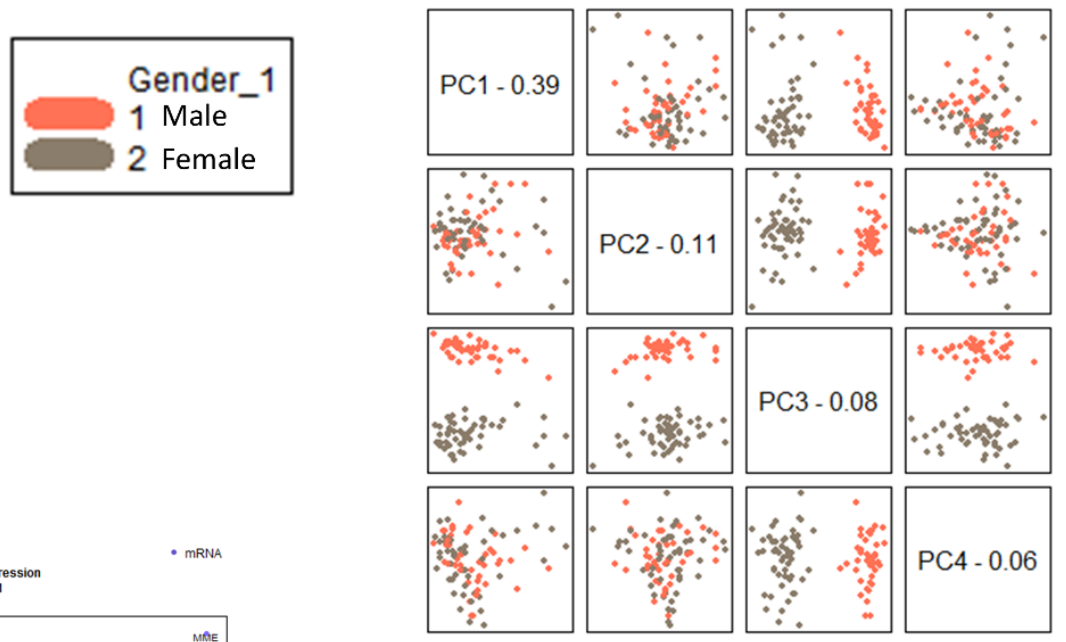

C. DE: Female vs. Male

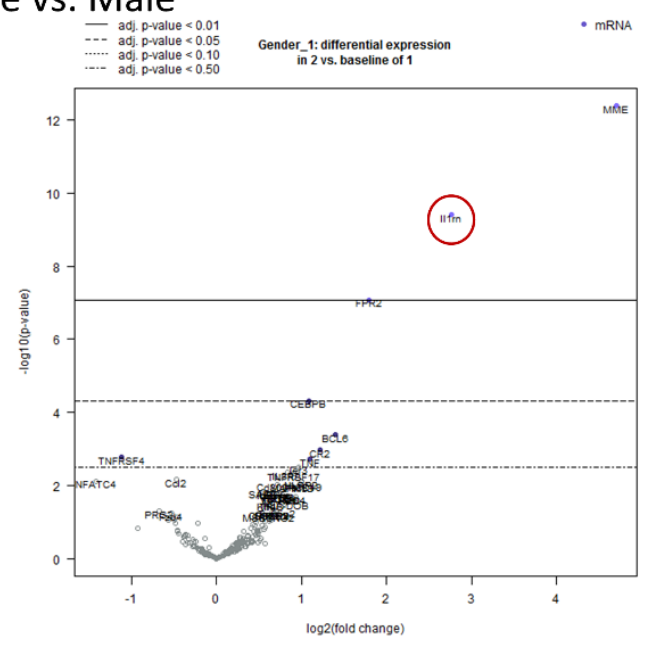

References

1. Manning, A.P., et al., Towards positive diagnosis of the irritable bowel. Br Med J, 1978. 2(6138): p. 653-4.

2. Ford, A.C., et al., Validation of the Rome III criteria for the diagnosis of irritable bowel syndrome in secondary care. Gastroenterology, 2013. 145(6): p. 1262-70 e1.

3. Drossman, D.A., et al., U.S. householder survey of functional gastrointestinal disorders. Prevalence, sociodemography, and health impact. Dig Dis Sci, 1993. 38(9): p. 1569-80.

4. Stone, A.A. and J.E. Broderick, Obesity and pain are associated in the United States. Obesity (Silver Spring), 2012. 20(7): p. 1491-5.

5. Cox, A.J., N.P. West, and A.W. Cripps, Obesity, inflammation, and the gut microbiota. Lancet Diabetes Endocrinol, 2015. 3(3): p. 207-15.

6. de Mello, V.D., et al., Gene expression of peripheral blood mononuclear cells as a tool in dietary intervention studies: What do we know so far? Mol Nutr Food Res, 2012. 56(7): p. 1160-72.

7. Robinson, J.M., et al., Complete blood count with differential: An effective diagnostic for IBS subtype in the context of BMI?. BioRxiv, 2019.

8. Robinson, J.M., AnalyzeBloodworkv1.0. 2019: GitHub. doi: https://doi.org/10.5281/zenodo.3373938.

9. Chatham, W.W. Glucocorticoid effects on the immune system. 2014; Available from: http://www.uptodate.com/contents/glucocorticoid-effects-on-the-immune-system.

10. Sasse, S.K. and A.N. Gerber, Feed-forward transcriptional programming by nuclear receptors: regulatory principles and therapeutic implications. Pharmacol Ther, 2015. 145: p. 85-91. 
11. Xavier, A.M., et al., Gene Expression Control by Glucocorticoid Receptors during Innate Immune Responses. Front Endocrinol (Lausanne), 2016. 7: p. 31.

12. Chinenov, Y., et al., Glucocorticoid receptor coordinates transcription factor-dominated regulatory network in macrophages. BMC Genomics, 2014. 15: p. 656.

13. Sevilla, L.M., et al., Glucocorticoid receptor and Klf4 co-regulate anti-inflammatory genes in keratinocytes. Mol Cell Endocrinol, 2015. 412: p. 281-9.

14. Abey, S.K., et al., Lysozyme association with circulating RNA, extracellular vesicles, and chronic stress. BBA Clin, 2017. 7: p. 23-35.

15. Geiss, G.K., et al., Direct multiplexed measurement of gene expression with color-coded probe pairs. Nat Biotechnol, 2008. 26(3): p. 317-25.

16. Nanostring, nCounter Advanced Analysis Plugin for nSolver Software User Manual, in MAN-10030-01. 2016, Nanostring Technologies, Inc.

17. Nanostring, nSolver 3.0 Analysis Software User Manual, in MAN-C0019-06. 2016, Nanostring Technologies: Seattle

18. Danaher, P., et al., Gene expression markers of Tumor Infiltrating Leukocytes. J Immunother Cancer, 2017. 5: p. 18.

19. Monaco, G., et al., RNA-Seq Signatures Normalized by mRNA Abundance Allow Absolute Deconvolution of Human Immune Cell Types. Cell Rep, 2019. 26(6): p. 1627-1640 e7.

20. Chaussabel, D., Assessment of immune status using blood transcriptomics and potential implications for global health. Semin Immunol, 2015. 27(1): p. 58-66.

21. $\mathrm{Li}, \mathrm{Y}$. and $\mathrm{X}$. Xie, A mixture model for expression deconvolution from RNA-seq in heterogeneous tissues. BMC Bioinformatics, 2013. 14 Suppl 5: p. S11.

22. Pollara, G., et al., Validation of Immune Cell Modules in Multicellular Transcriptomic Data. PLoS One, 2017. 12(1): p. e0169271.

23. Chen, B., et al., Profiling Tumor Infiltrating Immune Cells with CIBERSORT. Methods Mol Biol, 2018. 1711: p. 243-259. 